\title{
CÁLCULO DE PÉRDIDAS INDIRECTAS OCASIONADAS POR MOVIMIENTOS SÍSMICOS
}

\author{
Jaime García Pérez ${ }^{(1)}$ y Eric García López ${ }^{(2)}$
}

\begin{abstract}
RESUMEN
En el criterio de la minimización del costo total para la obtención de coeficientes de diseño sísmico, se consideran tanto costos iniciales como costos de las pérdidas ocasionadas por los temblores. En estos últimos se encuentran las pérdidas indirectas denominadas intangibles que incluyen vidas humanas. Este trabajo presenta métodos para calcular el valor que la sociedad está dispuesta a invertir para salvar estas vidas. Se analizan tanto los problemas individuales como sociales, poniendo énfasis en los primeros, donde el valor de la vida se puede inferir a partir de lo que la persona está dispuesta a aceptar o a pagar para reducir un riesgo a morir. Puesto que estos métodos emplean curvas de utilidad, se pone atención a ellas discutiendo los requisitos que deben cumplir. Asimismo, se discuten los casos del impacto personal, legados y herencias cuando se emplean las curvas de utilidad. Finalmente se presentan ejemplos de aplicación.
\end{abstract}

Palabras clave: costo total; coeficientes óptimos; intangibles; pérdidas; aceptación a pagar

\section{CALCULATION OF INDIRECT LOSSES DUE TO SEISMIC MOTIONS}

\begin{abstract}
In order to obtain seismic design coefficients, the total cost minimization criterion considers initial costs as well as costs of losses due to earthquakes. In the latter, we find indirect losses known as intangibles, which include human lives. This paper presents methods to calculate the value in which society should be willing to invest for saving lives. We analyze both individual and social problems. However, we pay attention to individual problems where the value of human life can be inferred by what the person should be willing to accept or to pay to reduce the risk of dying. As long as these methods use utility curves we pay special attention to them and discuss the requirements that they must meet. Moreover, the cases of personal impact, legacies and bequests when using utility curves are discussed. Finally, we present some examples.
\end{abstract}

Keywords: total cost; optimum coefficients; intangibles; losses; willingness to pay

\section{INTRODUCCIÓN}

Para optimar la confiabilidad en ingeniería sísmica tratamos de maximizar la utilidad asociada con el diseño del sistema en estudio. Esta utilidad considera el beneficio que resulta de la existencia del sistema, las pérdidas causadas por temblor incluyendo los daños que implican su posible falla, y su costo inicial. Entonces, nos proponemos seleccionar un parámetro de diseño sísmico de tal manera que se optimice una función objetivo (Rosenblueth 1964, Esteva 1968). Cuando calculamos las pérdidas debemos evaluar el valor de los intangibles como las vidas humanas. Pero en la práctica el ingeniero civil o decisor no puede

Artículo recibido el 29 de agosto de 2018 y aprobado para su publicación el 19 de junio de 2019. Se aceptarán comentarios y/o discusiones hasta cinco meses después de su publicación.

\footnotetext{
${ }^{1}$ Instituto de Ingeniería, Ingeniería Estructural, UNAM, Circuito Escolar, Coyoacán 04510, México DF. jgap@pumas.ii.unam.mx

${ }^{2}$ Instituto Nacional de Ciencias Penales, Magisterio Nacional 113, Tlalpan 14000, México DF. eric.garcia@inacipe.gob.mx
} 
encontrar la solución óptima ya que no existe un procedimiento formal y cuantitativo para calcular el elemento más importante que se pierde en caso de falla, las vidas humanas. Es necesaria una respuesta no importa que tan debatible o inaceptable pudiera parecer a los que juzguen al ingeniero o decisor. Esta circunstancia es lo que ha motivado el presente estudio.

En la literatura se encuentran varios métodos y técnicas que permiten obtener la solución óptima en la toma de decisiones racional, si se conoce la relación entre utilidad, por una parte, y por otra los beneficios, recursos, y pérdidas. En muchos problemas el tamaño de los beneficios, gastos y pérdidas son suficientemente pequeños y es válido suponer que la utilidad es una función lineal de ellos. Sin embargo, cuando se trata de vidas humanas, las pérdidas son generalmente tan altas que esta hipótesis no es válida y es necesario definir las formas de las curvas de utilidad. Los criterios propuestos en la literatura para valuar un intangible como la vida humana lleva a resultados tan distintos que no existe un criterio confiable.

El significado que se le da aquí al concepto valor de la vida humana es el de cuánto una persona o sociedad está dispuesta a invertir para preservar una vida. El valor sagrado de la vida en cualquiera de sus formas es una buena razón de ser. Sin embargo, cuando uno recibe un pago extra por realizar un trabajo bajo un riesgo, sin importar que tan pequeño es este riesgo, o cuando uno decide qué tan segura hacer una estructura, estamos asignando implícitamente un valor a la vida humana. Por lo tanto, el valor de la vida humana implica calcular varios valores. Por una parte, el valor de la vida propia, por otra el valor de una vida anónima, y por último el valor que la sociedad asigna a cada uno de sus integrantes. Los tres tipos de valores son diferentes y por tanto se determinan de manera distinta. Por todo lo anterior se deben de calcular de manera racional y de preferencia haciendo uso de la teoría de decisiones.

Los métodos principales que han estudiado el tema del valor de la vida humana son: El capital humano, el consumo y sus variaciones, el producto interno bruto, disposición a aceptar riesgos y disposición a evitarlos, los criterios de disposición combinados con el del capital humano, la consideración de posibles legados y herencias, las consideraciones de posibles seguros de vida, impacto social y personal, y la calidad de vida.

Estos métodos se revisan en un trabajo realizado por García-Pérez (2012), poniendo atención especial en el método del capital humano, donde el valor de una vida humana se toma como la esperanza del valor presente de la contribución de una persona al producto interno bruto, a través del resto de su vida. En el mismo trabajo, como una primera aproximación a la solución del problema considerando curvas de utilidad, se propone una forma sencilla de estas curvas con la que se calcula el valor que la sociedad estaría dispuesta a invertir para preservar una vida, normalizada por la cantidad que el criterio del capital humano asignaría a la vida del individuo. Se considera que el cociente así obtenido representa el factor por el que hay que multiplicar el resultado del criterio del capital humano, y así obtener el valor que una persona asignaría a su vida.

La objeción principal al método del capital humano y sus variaciones es que sólo se considera el lado económico del problema. Además, se considera la perfección del mercado del empleo y como sabemos éste no es así. Si se adopta la hipótesis de la perfección se distorsiona notablemente los resultados para los casos individuales. Es suficiente que exista el desempleo para que un trabajador sea remplazable, y algunas veces sin disminución de la producción o solo con una reducción parcial del mismo. Luego entonces el valor de algunas vidas toma el valor de cero, o sufre una reducción enorme si corregimos cómo se toma en cuenta el ingreso del desempleado, mientras que el valor de otras se reduce en mayor o menor extensión, dependiendo de la posibilidad de remplazo. Podríamos extendernos en señalar otras imperfecciones del mercado del empleo y sus consecuencias, pero no profundizaremos por ahora. 
Ya sea que el valor de una sola vida interese a la sociedad o al propietario de esa vida, debemos ignorar el método del capital humano y sus variaciones sin importar que tanto se usen o hayan sido usados. Debemos en cambio explorar la posibilidad de inferir el valor que cada quien asigne a su propia vida cuando se trata de enfrentar un determinado incremento en riesgo a cambio de una compensación económica, o de un gasto realizado para disminuir un riesgo específico de perder la vida. En este trabajo se adoptará el último criterio y puesto que emplea las curvas de utilidad, se pondrá atención especial a ellas. Además, procederemos de acuerdo con la teoría del utilitarismo. Todo eso se discute a continuación.

\section{PROBLEMAS INDIVIDUALES Y SOCIALES}

Tanto en los problemas de tipo individual como los de tipo social, se presenta el conflicto entre la escala de valores del decisor y la del cliente, ya sea que se trate de una persona o la sociedad. Desde el punto de vista normativo, podemos demandar que el decisor adopte la ética absoluta que consiste en maximizar la suma de las felicidades de todos los seres humanos de aquí a la eternidad (Rosenblueth 1987, 1992). En todos los casos, los criterios aplicados por el decisor será el resultado de negociaciones entre la ética absoluta y los intereses del cliente. En relación con los problemas individuales, la negociación se realiza entre la ética absoluta y la maximización de la esperanza del valor presente de la felicidad del individuo. Una combinación lineal de las felicidades de otros seres estará internalizada en esta felicidad. Por otra parte, los problemas de tipo social se negociarán con la esperanza del valor presente de la suma de las felicidades de los que conforman la sociedad, afectados del grado en que cada individuo pertenece a ella. Y una vez más, las felicidades de otros seres estarán internalizadas en una combinación lineal de ellas. En los problemas del primer tipo, el valor de una vida de un ser humano se infiere de lo que éste estaría dispuesto a pagar para reducir un riesgo de morir, o de qué riesgo estaría dispuesto a tomar a cambio de una compensación. Por otra parte, en los problemas del segundo tipo, la ética requiere que el decisor se coloque conceptualmente en las circunstancias de cada integrante de la sociedad.

\section{Valor individual de la vida}

Hablemos primero del caso de una persona que toma un riesgo de perder su vida en un tiempo corto y en un solo evento a cambio de una compensación. Definimos el valor de su vida como la relación de la compensación con la probabilidad de fallecer en una transacción que la persona haría si fuera racional. Inferimos el riesgo asignando un valor a la compensación y resolvemos para encontrar la probabilidad de fallecer que haría a la persona indiferente entre su situación actual y la transacción. Es decir, las utilidades esperadas en las dos situaciones deben coincidir (Howard 1979). Calculamos de manera similar el valor de la vida, infiriendo de una situación en la que el individuo está dispuesto a pagar una cantidad específica, a cambio de reducir la probabilidad de fallecer en corto tiempo. Definimos el valor de su vida como lo que está dispuesto a pagar dividido por la reducción de la probabilidad de fallecer, y otra vez inferimos las cantidades correspondientes igualando las utilidades en su situación actual y en caso de aceptar la transacción (Howard, 1979).

El valor individual de la vida depende de los conceptos siguientes: La curva de utilidad de la persona en términos de su ingreso o fortuna, la curva de utilidad de lo que representa para la persona los beneficios que sus herederos recibirán cuando ya no esté, el impacto personal de la perspectiva de fallecer y del proceso de fallecimiento en lo que respecta a la persona misma. Por lo que respecta a las utilidades en la situación actual, se debe considerar lo siguiente: La utilidad proveniente de factores económicos, la utilidad como la felicidad causada por factores no económicos, y la desutilidad proveniente de la angustia de la posibilidad de fallecer. 


\section{Valor social de la vida}

Desde el punto de vista de la sociedad, deseamos que la esperanza del valor presente de las utilidades de sus integrantes, en su situación actual, sea igual a la correspondiente a un segundo estado en el que la sociedad invierte recursos o recibe un beneficio a cambio de disminuir o aumentar, respectivamente, la probabilidad de fallecer de uno o más de sus integrantes. Si la posibilidad de que cada uno tiene de disfrutar los recursos de la sociedad, fuera independiente del número de habitantes, entonces la suposición de que el decisor debe proceder como si tuviera la misma probabilidad de colocarse en las circunstancias de cada integrante de la sociedad (con la probabilidad modificada por el grado de pertenencia) nos conduciría al valor de una vida anónima. Este valor sería el promedio de los valores individuales de todos los integrantes incrementado por el valor correspondiente del impacto social (Rosenblueth, 1987, 1992).

\section{UTILIDAD Y FELICIDAD}

La utilidad es la medida lógica de la intensidad de preferencia que satisface el axioma de von Neumann y Morgenstern (1943) que hace la utilidad sinónima con la utilidad esperada. Nos referiremos aquí a las preferencias en el sentido de lo que desearíamos moralmente preferir.

Hagamos que $>$ represente la relación de preferencia de tal manera que si $A$ y $B$ son dos estados posibles, entonces $A>B$ significa que $A$ se prefiere sobre $B$ para el individuo en estudio. Es posible entender "se prefiere" en un sentido descriptivo, como "el individuo se comporta como si él prefiere", en un sentido subjetivo como "el individuo siente como si él prefiere", o en el sentido normativo como "el individuo debe preferir". Aquí diremos que $A>B$ significa que el individuo prefiere $A$ sobre $B$, en el entendido de que la expresión simbólica acepta los tres significados. Ahora hagamos que $\prec \mathrm{y} \sim$ representen las relaciones de no preferencia y la indiferencia respectivamente. Es decir, $A \prec B$ significa que el individuo no prefiere $A$ sobre $B$, y $A \sim B$ que el individuo es indiferente entre estos dos estados.

Consideremos ahora un conjunto de $P$ estados posibles, $\left\{m_{i}\right\}, i=1, \ldots, p$. Supongamos que forman completamente un conjunto ordenado con respecto a la relación de preferencia. Esto significa que para toda $i$ y toda $j$, es necesario que $m_{i}>m_{j}$ o $m_{i} \prec m_{j}$. En otras palabras, $m_{i}>m_{j}, m_{i} \sim m_{j}$, y se cumple la relación de transitividad, es decir, si $m_{i}>m_{j}$ y $m_{j}>m_{k}$ entonces $m_{i}>m_{k}$. Bajo estas condiciones, $U_{i}$ es la utilidad del estado $m_{i}$ y $U_{j}$ la de $m_{j}$ si y solo si se cumplen dos condiciones. La primera es si $m_{i}>m_{j}$ es igual a $U_{i}>U_{j}$, y consecuentemente $m_{i} \prec m_{j}$ es igual a $U_{i}<U_{j}$ y $m_{i} \sim m_{j}$ es igual a $U_{i}=U_{j}$. La segunda si $m$ significa que los estados $m_{1}, \ldots, m_{p}$ pueden ocurrir con respectivas probabilidades $Q_{1}, \ldots, Q_{p}$ entonces la utilidad de $m$ es $U=E\left[U_{i}\right]=\sum_{i}^{p} Q_{i} U_{i}$ donde $E$ representa el valor esperado.

Para asignar valores a la utilidad, podemos proceder de la manera siguiente: primero reordenamos los estados $m_{i}$ de tal forma que la intensidad de preferencia es no decreciente con $i$, y $m_{1} \prec m_{2} \ldots \prec m_{p}$. Si $m_{1} \sim m_{p}$ asignamos cualquier valor a todas las $U_{i}$ independiente de $i$. Si $m_{p}>m_{1}$, asignamos valores arbitrarios a $U_{1}$ y $U_{p}$ dependiendo solamente de que $U_{1}<U_{p}$. Entonces presentamos al individuo con la posibilidad de participar en una lotería $I$, en la que con una probabilidad $R$, estará en el estado $m_{1}$ y con probabilidad $1-R$ en el estado $m_{p}$, y le preguntamos al individuo que exprese su preferencia entre el estado $m_{i}$ y la lotería. Enseguida variamos $R$ hasta $m_{i} \sim I$. Si hacemos que $R_{i}$ sea el valor de $R$ por el que la el individuo es indiferente entre $m_{i}$ y la lotería. Entonces, $U_{i}=R_{i} U_{1}+\left(1-R_{i}\right) U_{p}$, ya que la utilidad de $I$ es el Segundo miembro de esta ecuación de acuerdo con el axioma de von Neumann y Morgenstern. Por otro lado, la utilidad está definida exclusivamente por una transformación lineal positiva, la que es evidente 
cuando escogemos $U_{1}$ y $U_{p}$ arbitrariamente sin mayor condición que $U_{p}>U_{1}$ si $m_{p}>m_{1}$ o $U_{p}=U_{1}$ si $m_{p} \sim m_{1}$.

La utilidad en el sentido ordinario es una medida a priori, es decir, antes de la experiencia de los estados a los cuales se refiere. La felicidad en un sentido cuantitativo es una utilidad a posteriori. Es posterior a la experiencia de los estados, a los que se refiere, y bajo la hipótesis de que la persona recuerda sus experiencias perfectamente. En la teoría de decisiones, el valor esperado de la felicidad es una utilidad preposterior, en otras palabras, una medida de la intensidad de preferencia que una persona tendría si hubiera experimentado los estados en estudio, siendo evaluada antes de que los experimente. La utilidad mide la intensidad de deseo; la felicidad la intensidad como se hubiera preferido haber experimentado un estado, y en teoría de decisiones, el valor esperado de la felicidad es el valor esperado de la intensidad, como el individuo hubiera preferido haber experimentado.

\section{CURVAS DE UTILIDAD}

El cálculo de un intangible como el valor de la vida humana requiere conocimiento de las formas de las curvas de utilidad en función de la riqueza e ingreso de los individuos en cuya vida estamos interesados. Cuando damos a la utilidad su significado habitual de intensidad de deseo, o el de preferencia a priori, estas curvas deben cumplir ciertas condiciones (Keeney y Raiffa, 1976; Rosenblueth 1987, 1992). Estas condiciones aplican a la utilidad en función de la riqueza total incluyendo la esperanza del valor presente de ingresos futuros. Expresaremos estas curvas como $U=U(W)$, es decir la utilidad de la riqueza $W$, y $W_{\min }$ como el mínimo valor de $W$ para sobrevivir. Además, $W$ y $U$ son las esperanzas del valor presente de la riqueza y la utilidad respectivamente. Las condiciones mencionadas son:

$1 U(W)=0$ si $W<W_{\min }$. Esto implica que la utilidad de una persona que deja de existir es nula. Se impone arbitrariamente y con esto aceptamos ya sea que $W$ incluye la riqueza total o que no hay seguro de desempleo.

$2 U^{\prime}(W)>0$ si $W \geq W_{\text {min }}$, donde la prima indica la derivada con respecto de $W$. Si alguien no desea recibir una cantidad de dinero, puede donar el excedente y permanecer como antes.

$3 U^{\prime \prime}(W)<0$ si $W \geq W_{\text {min }}$, es decir, $U(W)$ es cóncava. Las personas emplean usualmente los primeros ingresos para cubrir las necesidades más urgentes, luego entonces éstas son las de mayor valor, y generalmente, el valor de los ingresos disminuye cuando la riqueza aumenta.

$4 U^{\prime \prime 2}(W)<U^{\prime}(W) U^{\prime \prime \prime}(W)$ si $W \geq W_{\min }$. Esto equivale a decir que la aversión al riesgo, definida como $-U^{\prime \prime}(W) / U^{\prime}(W)$, debe ser una función decreciente de $W$. Un individuo, que con una cierta riqueza está dispuesto a aceptar ciertos riesgos, debería estar dispuesto a los mismos riesgos y a más con una mayor riqueza.

$5 U(\infty)=U_{\max }<\infty$. Esta condición resulta de la finitud de los seres humanos. Somos capaces de desear con una intensidad finita. Es costumbre asignar el valor de la unidad al valor máximo posible.

$6 U(W)>0$ si $W \geq W_{\min }$. Aunque la miseria de algunas personas es tal que desearían dejar de existir, lo opuesto también es verdad.

\section{Funciones}

En la literatura se encuentran algunas funciones de utilidad con diferentes formas tales como la exponencial, la logarítmica y la cuadrática. Keeney y Raiffa (1976) y Howard (1979) han propuesto algunas funciones de utilidad para resolver el problema. En este trabajo, con base en las funciones de dichos autores y con pequeños cambios en los parámetros, emplearemos dos funciones de utilidad que cumplen con los 
requisitos mencionados en la sección anterior. La primera función se muestra en la figura 1 y está dada por la siguiente expresión:

$$
U(W)=\left(1-\alpha_{1} e^{-n_{1} \delta}\right) U_{\max }
$$

Donde $\alpha_{1}$ y $n_{1}$ son constantes, y los valores de $\alpha_{1}$ se encuentran entre cero y uno, $U_{\max }$ es la utilidad máxima posible, suponiendo que no tenemos ninguna restricción económica, y $\delta=\left(W-W_{\min }\right) / W_{\min }$ es la riqueza neta normalizada. Se ilustra con dos curvas. La de línea continua se grafica con $\alpha_{1}=1$, y la punteada con $\alpha_{1}=0.5$, y ambas gráficas con $n_{1}=0.1$. La segunda función se presenta en la figura 2 y está dada por:

$U(W)=\left(1-\alpha e^{-a \delta}-\beta e^{-b \delta}\right) U_{\max }$

aquí $\alpha, \beta, a, \mathrm{y} b$ son constantes. con $\alpha+\beta \leq 1, \mathrm{y} \alpha \neq \beta$. La línea continua emplea $\alpha=0.1, \beta=0.9, \mathrm{y}$ los valores para la gráfica con línea punteada son $\alpha_{1}=0.1, \beta=0.4$. Ambas gráficas emplean $a=0.01, \mathrm{y}$ $b=0.18$. La ec. 1 es un caso particular de la ec. 2 correspondiente a: $a$ o $b=0$ o $\infty, \alpha o \beta=0$, o $a=b$.

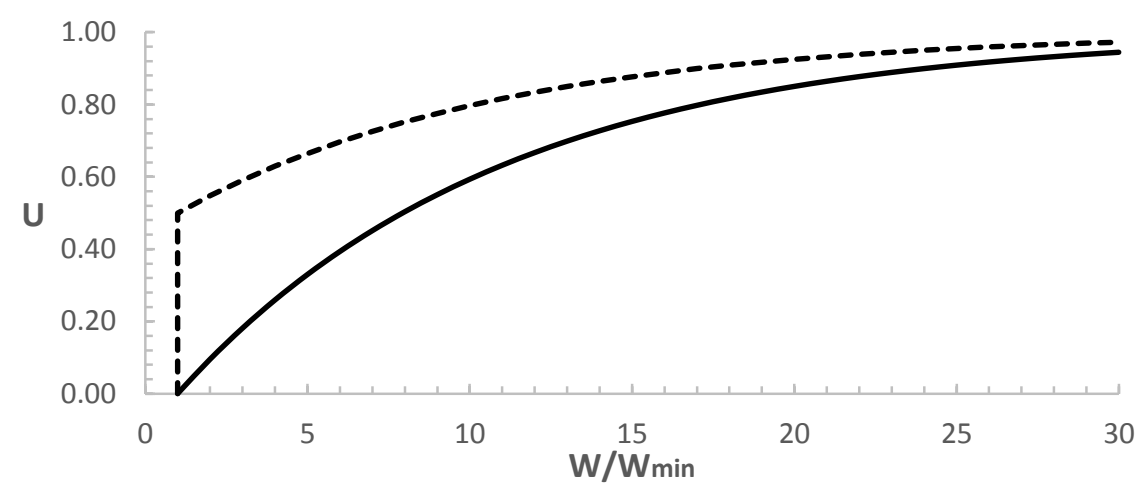

Figura 1. Primera función de utilidad con $U_{\min }=0$ (continua) y 0.5 (punteada)

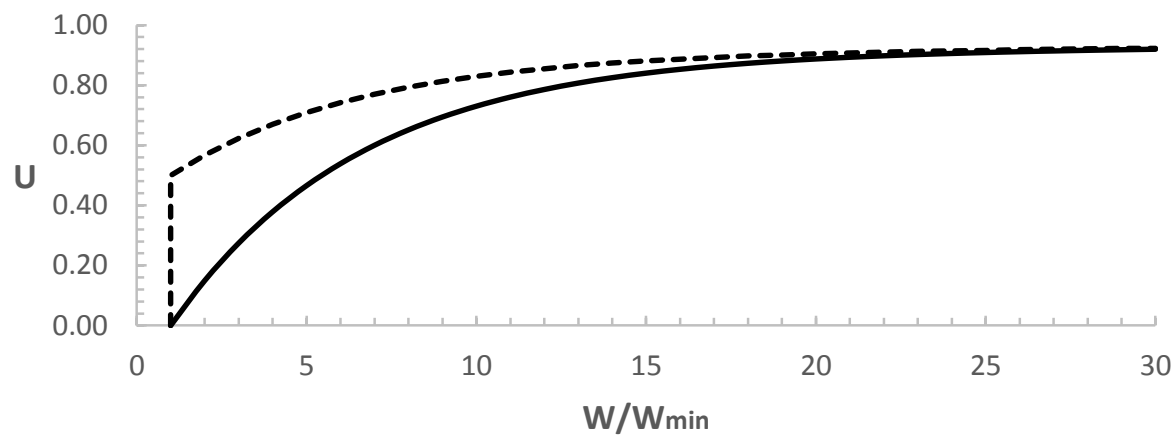

Figura 2. Segunda función de utilidad con $U_{\min }=0$ (continua) y 0.5 (punteada)

En las figuras 1 y 2 las líneas continuas representan los casos cuando $U_{\min }=0$ que significa que cuando el ingreso es tan pequeño que la persona apenas sobrevive, es incapaz de disfrutar en absoluto. Sin embargo, el conocimiento actual acepta la hipótesis que, excepto en la vecindad de $W_{\min }, U$ debe ser finita y no trivial. Es bien conocido que los valores no económicos son más preciados que los económicos. Las líneas punteadas representan los casos cuando $U_{\min }=0.5 \mathrm{U}$ a partir de los resultados encontrados por 
Rosenblueth (1987). Ambas figuras tienen como límite superior $U_{\max }=1 \mathrm{y}$ comienzan a partir de $W / W_{\min }=1$.

La concavidad de las figuras anteriores toma en cuenta la aversión al riesgo que se menciona en el punto 4 de las condiciones de estas curvas. En estas figuras se ha optado por presentar normalizado el valor de $W$ con respecto del valor mínimo para sobrevivir y que $U_{\min }=U\left(W_{\min }\right)$. Si aceptamos que las utilidades son aditivas, $U_{\min }$ es la utilidad que proviene de conceptos no económicos, como una fracción de la utilidad máxima posible $U_{\max }$ (que en este caso se ha tomado como el valor de uno), cuando la persona no está ante la perspectiva inmediata de fallecer, ni de que fallezcan sus seres queridos. Entonces $U_{\min }$ es la utilidad de disfrutar el hecho de estar vivo, de disfrutar experiencias culturales, de contemplar la lluvia, de tener buenos amigos. Actualmente se está trabajando para plantear métodos objetivos para la evaluación cuantitativa de esta utilidad.

\section{CRITERIOS DE DISPOSICIÓN SIN LEGADOS O HERENCIAS}

Estos criterios tratan de responder a la pregunta "¿No sería bueno preguntar a los involucrados en cuánto valoran ellos su vida? Planteando de esta manera el problema es posible deducir el valor que cada uno asigna a su vida cuando enfrenta un incremento en el riesgo a cambio de una compensación económica, o de un gasto hecho para disminuir un riesgo específico de perder la vida.

\section{Disposición a aceptar}

Howard(1979) explica este método como sigue: Digamos que ofrecemos a una persona una pastilla de color negro, advirtiéndole que si la ingiere, tiene la probabilidad $F$ de perder la vida en corto tiempo. Enseguida le preguntamos por cual cantidad de dinero estaría dispuesta a ingerir la pastilla. Suponemos que la persona responde que por la cantidad $E$. Entonces, razonamos que la persona actúa como si el valor de su vida para ella fuera $L=E / F$.

Consideremos ahora una persona cuya curva de utilidad es conocida, le preguntamos qué compensación económica requeriría para estar dispuesta a tomar un riesgo específico de perder la vida. Hagamos que $U(W)$ represente la utilidad asociada a la esperanza del valor presente de su ingreso futuro y que $E$ sea la compensación que requeriría para emprender una actividad con probabilidad $F$ de perder la vida. La suma no debería ser menor que la de una situación de indiferencia entre su situación actual y la asociada con el riesgo y compensación (Howard, 1979), luego entonces podemos escribir $U(W)=$ $(1-F) U(W+E)$. Sin importar la expresión de $U(W)$, podemos asignar valores a $E$ y calcular la $F$ correspondiente. Luego de conocer estas dos variables podemos calcular el valor de la vida en estas circunstancias como $L=E / F$.

\section{Disposición a pagar}

Howard (1979) pregunta ahora por la respuesta al problema de la pastilla blanca, es decir, cuánto está dispuesta una persona a recibir como pago por ingerir una pastilla que elimina la probabilidad $F$ de que la persona hubiera perdido la vida en corto tiempo. El método es similar al caso de disposición a aceptar con $(1-F) U(W)=U(W-E)$ y la expresión $L=E / F$ es aun válida. 


\section{Disposición para riesgos pequeños}

Cuando lidiamos con riesgos pequeños ambo métodos anteriores son indiferentes y el límite de $L$ cuando $F$ tiende a cero está dada por la siguiente expresión (Howard 1979, Rosenblueth 1987, 1992).

$L=U / U^{\prime}$

Se ha presentado la herramienta para tratar con problemas en el que la probabilidad de perder la vida es grande. En casi todos los problemas en que estamos interesados, esa probabilidad es muy pequeña, y las tasas de mortalidad están entre $10^{-5}$ y $10^{-7}$ por año (Starr 1969, Starr y Whipple 1980). Luego entonces la ec. 3 puede aplicarse con precisión, y la emplearemos para ilustrar los criterios de disposición en los ejemplos que se muestran.

\section{CRITERIOS DE DISPOSICIÓN CONSIDERANDO LEGADOS O HERENCIAS}

Una de las principales razones por la que una persona ahorra es para tratar de cubrir las incertidumbres en sus ingresos. El deseo de heredar a parientes cercanos o a una causa en la que la persona considere merecedora, explica solo una parte de la costumbre de ahorrar. Por lo tanto, consideraremos la cantidad ahorrada como un dato y no tratar de inferirla de las curvas de utilidad.

Hay dos tipos de problemas en los que estamos interesados en distinguir entre la utilidad de lo que una persona consume y la que está asociada a lo que ella ahorra y que dejará a sus herederos. En el primer tipo, se trata de un solo evento que ocurrirá en un tiempo corto y en el que la persona puede perder su vida. En el segundo tipo, la probabilidad de perder la vida por unidad de tiempo se ve afectada, es decir, la tasa de mortalidad es relevante a la persona. En problemas del primer tipo, la persona no tiene tiempo de modificar su consumo a menos de que sobreviva. Si la persona está dispuesta a correr el riesgo a cambio de cierta compensación, esta se asignará necesariamente a sus herederos en caso de que ella pierda la vida.

En el caso de legados o herencias, la utilidad total es la esperanza de lo que la persona asigna a la condición en la que sobrevive al riesgo con aquella la que deja de existir, determinándose esta última por la utilidad de que a la persona en estudio representa el hecho de que sus herederos disfruten su riqueza. Si la riqueza de la persona es $W$ teniendo el dilema de ingerir la pastilla de color negro, con probabilidad $F$ de perder la vida a cambio de cierta compensación o rechazar la oferta, su utilidad está dada por $U(W)=$ $(1-F) U_{A}(W)+F U_{D}(W)$, donde $U_{A}(W)$ y $U_{D}(W)$ son las utilidades correspondientes a los estados de vivir o dejar de existir. A partir de la ec. 3 podemos escribir que el valor de la vida humana para cambios pequeños en riesgo está dado por $L=\left(U_{A}-U_{D}\right) /(1-F) U_{A}^{\prime}(W)+F U_{D}^{\prime}(W)$. De aquí se puede observar que debido a la resta en el numerador y a la incorporación del término $F U_{D}^{\prime}(W)$ en el denominador, esta expresión da valores menores que cuando no se consideran legados o herencias. En este momento no se cuenta con estudios para definir las formas de las curvas de las utilidades $U_{D}$ ni para extender los resultados a condiciones de aumento o disminución en las tasas de mortalidad.

\section{IMPACTO PERSONAL}

Cuando una persona deja de existir, no pierde solamente su valor de $U$, sino que también se presenta una desutilidad adicional, se le ha denominado a este valor como impacto personal (Rosenblueth 1987). Sin este impacto, podemos estar seguros que la humanidad no existiría, puesto que casi todos pasamos por estados depresivos en los que uno preferiría no existir, pero encuentra algo que previene a uno de perder la vida. Por otra parte, si la persona no pierde la vida debido a la decisión, algún día fallece de lo que llamamos 
muerte natural, lo que trae una pérdida diferida por impacto personal. El impacto personal neto $I_{p}$ se obtiene de substraer el impacto debido a muerte natural del impacto personal, y se ha encontrado que es aproximadamente $U_{\min }$ (Rosenblueth 1987). Por lo tanto, en términos de la esperanza del valor presente de la utilidad, cuando una persona deja de existir implica una pérdida total de $U+I_{p}$ para ella. El valor de la vida humana para un individuo cuando tiene la opción de recibir una compensación a cambio de tomar un pequeño incremento en riesgos está dado por $L=\left(U+I_{p}\right) / U^{\prime}$ (Rosenblueth 1987). La figura 3 muestra el impacto personal en una curva de utilidad.

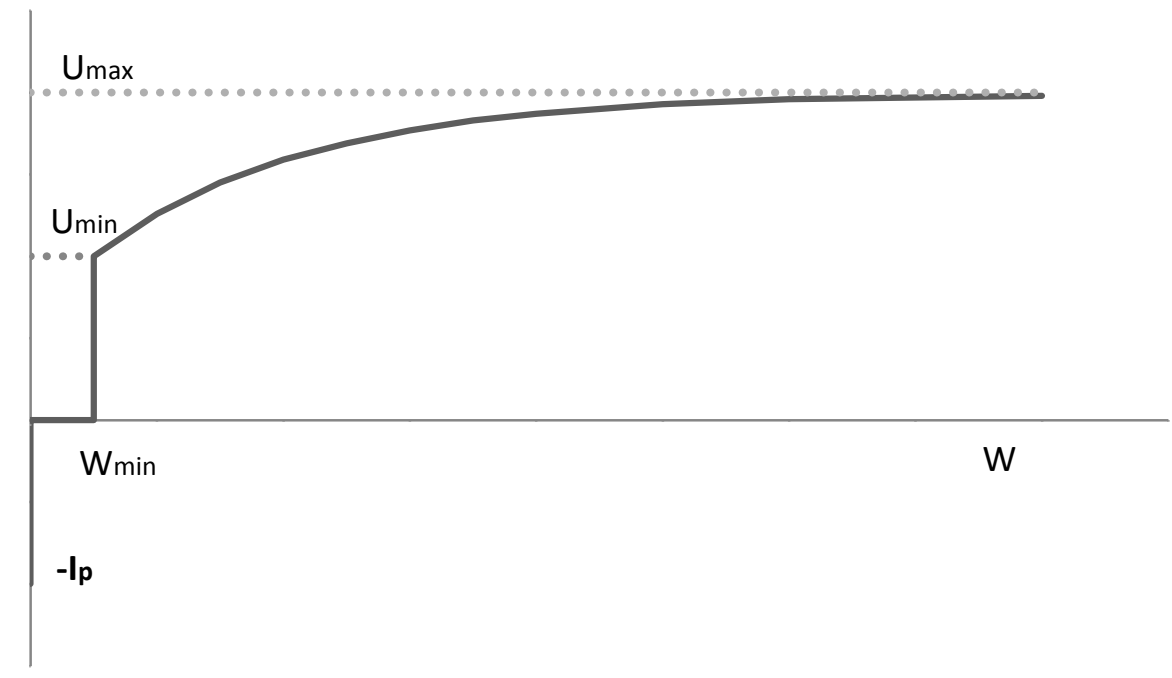

Figura 3. Impacto personal en la curva de utilidad

La magnitud del impacto depende significativamente de la naturaleza exacta de la actividad peligrosa por la que una persona se encuentra en riesgo, es decir, de la causa inmediata de fallecimiento. Una aversión excesiva a alguna de estas actividades prevalece como el cáncer, las plantas nucleares o los viajes en avión. Existe una indiferencia relativa a otras como los accidentes de tránsito. Por lo tanto, el decisor debería inclinarse a eliminar estas diferencias, haciendo claras sus intenciones, pero no puede ignorarlas.

\section{APLICACIONES}

\section{Funciones de utilidad}

Si transformamos la utilidad y la esperanza del valor presente del ingreso por sus valores por unidad de tiempo, podemos aplicar las ecs. 1 y 2 para resolver algunas preguntas tales como cuánto estaría dispuesta una persona con ingreso mensual de $w$ dólares a invertir en un negocio que, de resultar exitoso, le proporcione un ingreso adicional de $j$ dólares mensuales a cambio de una inversión de $g$ dólares mensuales. De otra forma la persona perdería solamente su inversión con una probabilidad de 0.5 . La cantidad que la persona estaría dispuesta a invertir puede obtenerse igualando su utilidad actual con la esperanza de las utilidades que resultarían de los dos estados posibles originados por la inversión. Entonces, podemos escribir:

$U(W)=0.5[U(w+f-g)+U(w-g)]$

Sustituyendo la ec. 1 en la 4 encontramos que $e^{-n_{1} k}=e^{-n_{1} d}+1$,donde $k=g / w_{\text {min }}, d=j / w_{\text {min }}$, y en que $w_{\min }$ es la cantidad mínima de ingreso familiar para sobrevivir. De aquí podemos saber el máximo 
valor de $k$ dado por $k=\frac{1}{n_{1}} \ln \left[2 /\left(e^{-n_{1} d}+1\right)\right]$. La figura 4 muestra una gráfica con los valores de inversión $k$ en términos del ingreso adicional $d$.

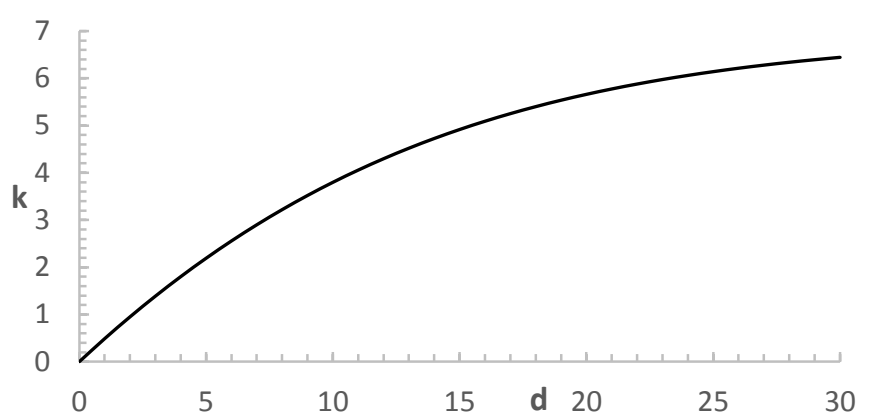

Figura 4. Ingreso adicional e inversión empleando la primera curva de utilidad

Siguiendo el mismo procedimiento empleando la segunda curva de utilidad, sustituyendo la ec. 2 en la 4 encontramos que $\alpha e^{-a \delta}\left[-2+e^{a k}\left(e^{-a d}+1\right)\right]=-\beta e^{-b \delta}\left[-2+e^{b k}\left(e^{-b d}+1\right)\right]$, y otra vez calculamos el valor máximo de $k$, empleando $\alpha=0.1, \beta=0.4$ y $\delta=10$. La figura 5 muestra la gráfica correspondiente.

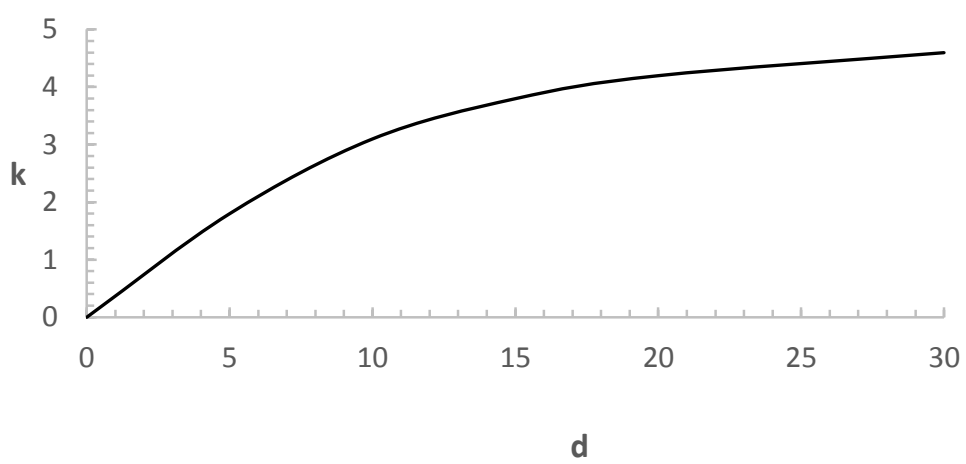

Figura 5. Ingreso adicional e inversión empleando la segunda curva de utilidad

En ambos casos presentados debemos establecer un límite superior para el valor máximo de $k$ de tal manera que $k \leq \delta$, ya que la persona no invertiría más allá de su ingreso neto (eliminando del total aquellos que la persona y su familia necesitarían). Observamos en las figuras 4 y 5 que las formas de las curvas para ambos modelos son similares y que los valores máximos están cercanos a 7 y 5 respectivamente para este ejemplo. Si contamos con datos empíricos, los resultados obtenidos con las curvas deben coincidir con los resultados empíricos, de otra forma ensayaríamos nuevos valores de los parámetros y repetiríamos el análisis hasta reconciliar los resultados.

\section{Criterios de disposición}

Empleando la curva de utilidad dada por la ec. 1 con $\alpha_{1}=0.5$, y $n_{1}=0.1$, calculamos el valor de $L$ a partir de la ec. 3. La figura 6 muestra el factor $f=L / W$ para diferentes valores de $W / W_{\text {min }}$. Si el método del capital humano asigna el valor de $W$ a la vida humana, el factor $f$ sería el factor por el cual uno podría multiplicar para calcular el valor que un individuo asignaría a su vida. Este factor siempre es mayor que 1. 


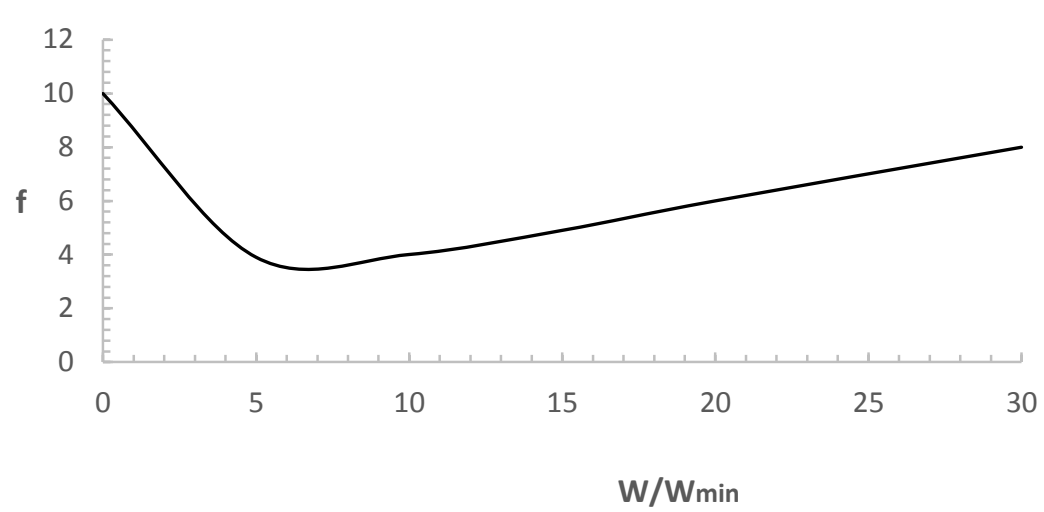

Figura 6. Factor para el criterio de disposición para riesgos pequeños empleando la primera curva de utilidad

Empleando la curva de utilidad proporcionada por la ec. 2 con $\alpha=0.1, \beta=0.4, a=0.01$, y $b=0.18$, calculamos el valor de $L$ empleando la ec. 3. La figura 7 muestra el factor $f=L / W$ para diferentes valores de $W / W_{\text {min }}$, con el mismo significado que para el caso anterior.

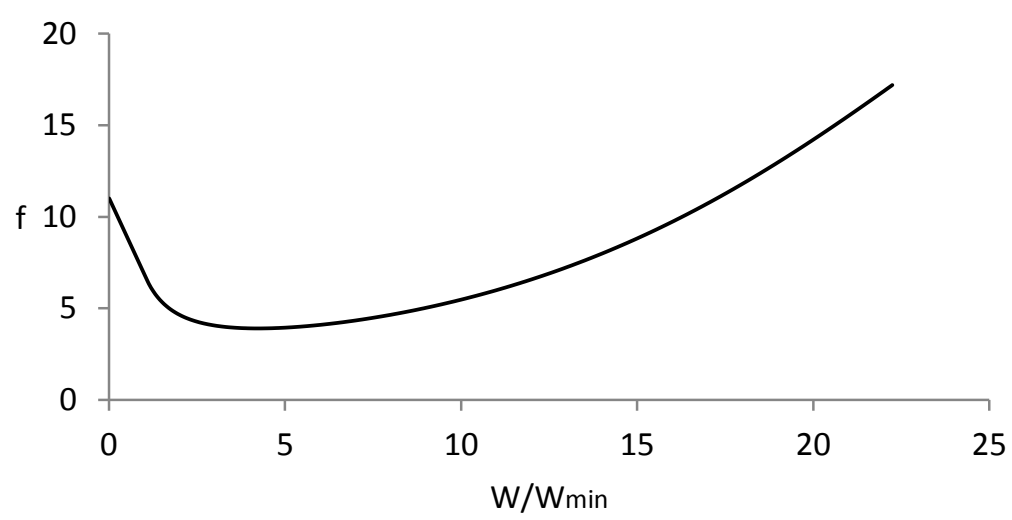

Figura 7. Factor para el criterio de disposición para riesgos pequeños empleando la segunda curva de utilidad

\section{Coeficientes de diseño sísmico óptimos}

El objetivo principal de este artículo es el de presentar métodos para calcular el valor que está dispuesta a invertir la sociedad para preservar una vida. Posteriormente se tiene el reto de incorporar este valor al proceso de la selección de parámetros de diseño. Se necesita una discusión a fondo en cómo el método desarrollado en este trabajo puede mejorar las actuales iniciativas del diseño sísmico (ingeniería sísmica con base en el desempeño) y así promover una discusión para implementaciones prácticas. Esto está fuera del alcance de este trabajo. En aras de ilustrar la aplicación de los resultados obtenidos, se empleará aquí una regla de decisión sencilla, que incluye el valor calculado de lo que está dispuesta a invertir la sociedad para preservar una vida, como un valor aditivo en las pérdidas en la formación de la función objetivo. Esto se explica a continuación.

El método que se empleará para calcular los parámetros de diseño sísmico óptimo lo propusieron por primera vez Rosenblueth (1964) y Esteva (1968). Estos autores consideran que el proceso de ocurrencia de 
los temblores es Poisson, que el costo inicial de una estructura y el costo debido a futuros temblores dependen solamente de la medida de la intensidad, y que el sistema se reconstruye inmediatamente después de cada falla. El método considera la optimación de la esperanza del valor presente del costo total $z(c)$, que incluye el costo inicial $x(c)$ de las estructuras, y la esperanza del valor presente del costo debido a temblores futuros $y(c)$. Aquí $c$ representa el coeficiente de diseño sísmico. Entonces, se desea optimar la siguiente función objetivo.

$$
z(c)=x(c)+y(c)
$$

Con base en los trabajos realizados por Whitman y col (1973), Grandori (1977), Ferrito (1984), Rosenblueth (1987), y Vargas y Jara (1989), es razonable adoptar la expresión siguiente para el costo inicial de las estructuras.

$x(c)= \begin{cases}C_{1} & \text { if } c \leq c_{0} \\ {\left[1+\alpha_{2}\left(c-c_{0}\right)^{\alpha_{3}}\right] C_{1}} & \text { if } c>c_{0}\end{cases}$

Donde, si la estructura no se diseña para resistir temblores, $C_{1}$ sería su costo correspondiente y $c$ sería su resistencia lateral, $\alpha_{2}$ y $\alpha_{3}$ son constantes.

La esperanza del valor presente del costo debido a temblores futuros $y(c)$ puede calcularse como (Esteva 1968, Rosenblueth 1976a, Rackwitz 2000)

$y(c)=H(c) v(c) / \gamma$

Donde $v(c)$ es la tasa de excedencia de una demanda sísmica, $\gamma$ es la tasa de descuento que representa el valor del dinero en el futuro, y $H(c)$ es la esperanza del valor presente de las pérdidas cada vez que ocurre un temblor. Estas pérdidas son las consecuencias de la falla de la estructura más allá de su propio costo de construcción, y está dada por $x(c)+s(c)$ (Rosenblueth 1976b). En la mayoría de los casos $s(c)$ será constante, incluyendo el costo directo del daño físico y el costo de la demolición y remoción, así como el costo de vidas humanas y lesiones de tal forma que $s(c)=s$ puede emplearse.

Substituyendo las ecs. 6 y 7 en la ec. 5 y normalizando con respecto de $C_{1}$ obtenemos la esperanza del valor presente que debe optimarse, dada por la siguiente expresión.

$\frac{z(c)}{C_{1}}=1+\alpha_{2}\left(c-c_{0}\right)^{\alpha_{3}}+\left[1+\alpha_{2}\left(c-c_{0}\right)^{\alpha_{3}}+\frac{s}{C_{1}}\right] v(c) / \gamma$

La aplicación de la ec. 8 se ilustra a través de un ejemplo en el que se calcula el coeficiente de diseño sísmico óptimo para un sitio con una tasa de excedencia dada por $v(c)=(0.001 / c)^{r}$, donde $r$ depende de la ubicación del sitio en estudio, y toma el valor de 1.5 para un sitio de baja sismicidad (Ordaz y col 2017). Empleamos además $\alpha_{2}=0.5, \alpha_{3}=1.3, c_{0}=0.05, C_{1}=10^{5}$ (García-Pérez y col 2005, García-Pérez 2016). La tabla 1 presenta diferentes coeficientes de diseño sísmico óptimos para tres casos donde la riqueza $W$ es 1,5 y 15 veces el valor mínimo necesario para sobrevivir $W_{\min }$.

El valor correspondiente de $s$ considera solamente el costo de vida humana y se calcula tomando el valor del capital humano de 45000 dólares obtenido en García-Pérez (2012) empleando datos para México, posteriormente se multiplica por el factor $f$ de la figura 7 correspondiente a cada $W / W_{\text {min }}$. Es importante recordar que las curvas de utilidad propuestas en conjunto con sus derivadas se emplearon para calcular el valor de $f$. Esto es una manera aproximada de la aplicación de los resultados obtenidos en este trabajo. Una vez obtenido el valor de $s$ se calcula el valor mínimo de los coeficientes empleando la ec. 8. Cabe hacer 
notar que debido a que nos interesa el mínimo y no los costos puede emplearse si ningún problema en la forma normalizada que se indica. Los valores óptimos para los coeficientes de diseño sísmico se consignan en la última columna.

Tabla 1. Coeficientes de diseño sísmico óptimos

\begin{tabular}{cccc}
\hline$W / W_{\min }$ & $f$ & $s\left(10^{5}\right)$ & $c_{\text {opt }}$ \\
\hline 1 & 6.85 & 3.1 & 0.17 \\
5 & 3.94 & 1.8 & 0.15 \\
15 & 8.81 & 4.0 & 0.18 \\
\hline
\end{tabular}

En este ejemplo se ha intentado dar una respuesta parcial en cómo el costo de salvar vidas puede emplearse para la selección de coeficientes. A pesar de lo simple del ejemplo, se observa que los valores están de acuerdo con los obtenidos en otros estudios (Ordaz y col 2017). Sin embargo, la incorporación del costo de salvar vidas en la función objetivo, requiere un estudio profundo.

\section{COMENTARIOS FINALES}

En este trabajo nos interesamos por contestar la pregunta a cuánto está dispuesta la sociedad a invertir para salvar vidas. Para esto, discutimos conceptos de la teoría de utilidades, felicidad, valores de la vida individual y social, e impacto personal. Se estudiaron dos curvas de utilidad en función de la riqueza de un individuo. A partir de estas curvas fuimos capaces de estimar el valor de la vida humana cuando se trata con riesgos pequeños. Se realizó una aplicación para el cálculo de coeficientes de diseño sísmico óptimos en un sitio de baja sismicidad. Se tiene que aprender más acerca del valor tanto individual como social de la vida humana, especialmente en lo relacionado con la selección de las curvas de utilidad. Estas curvas son función de varias variables incluso para una misma persona, aun en circunstancias externas estacionarias, evolucionan con el tiempo. Es necesario definir exactamente tanto las formas como las ordenadas máximas de dichas curvas de utilidad. También es importante establecer las utilidades provenientes de factores no económicos, en particular, la alegría de estar vivo. Es necesario llevar a cabo encuestas sobre preferencias entre diferentes alternativas y loterías de tipo conceptual, así como ejercicios Delfos; de tal manera que se puedan establecer los valores de los diferentes parámetros que gobiernan las curvas de utilidad.

\section{REFERENCIAS}

Esteva, L (1968), "Bases para la formulación de diseño sísmico", tesis doctoral, Facultad de Ingeniería, UNAM.

Ferrito, J M (1984), "Economics of seismic design for new buildings", Journal of Structural Engineering, ASCE; 110 (12): 2925-37.DOI:10.1061/(ASCE)0733-9445(1984)110:12(2925)

García-Pérez, J, F Castellanos y O Díaz (2005), "Occupancy importance factor in earthquake engineering”, Engineering Structures, 27, 1625-32. DOI: 10.1016/j.engstruct.2005.05.017

García-Pérez, J (2012). "The value of risk reduction in optimum seismic design", 15th World Conference of Earthquake Engineering, Lisbon, Portugal.

García-Pérez, J (2016). "Expected present value of losses in the computation of optimum seismic design parameters", International Journal of Geological and Environmental Engineering. Publication, 10004524. 10, 6, 626-631.

Grandori, G (1977), "Seismic zoning as a problem of optimization", Proceedings of the $2^{\text {nd }}$ international conference on structural safety and reliability, p. 613-24. 
Howard, RA (1979), "Life and death decision analysis" Research Report No. EES DA-79-2, Department of Engineering-Economic Systems, Stanford University, Stanford, California 94305.

Keeney, RL y H Raiffa (1976). "Decisions with multiple objectives: preferences and value trade-offs", John Wiley \& sons, New York.

Ordaz, M, A Salgado-Gálvez, LE Pérez-Rocha, O Cardona, y U Mena-Hernández (2017). "Optimum earthquake design coefficients based on probabilistic seismic hazard analyses". Theory and applications, Earthquake Spectra, 33(4), DOI: 10.1193/110116EQS189M

Rackwitz, R (2000), "Optimization- The basis of code-making and reliability verification", Structural Safety, 22, 27-60, DOI: 10.1016/S0167-4730(99)00037-5

Rosenblueth, E (1964), "Closure to probabilistic design to resist earthquakes", Journal of the Engineering Mechanics Div. Proc. ASCE, 90, EM5.

Rosenblueth, E (1976a), "Optimum design for infrequent disturbances". Journal of the Structural

Division ST9, 1807-1825.

Rosenblueth, E (1976b); “Towards optimum design through building codes". Journal of the Structural Division ST3, 591-607.

Rosenblueth, E (1987), "What should we do with structural reliabilities", Reliability and risk analysis in civil engineering. Proceedings of the fifth international conference on applications of statistics and probability in soil and structural engineering.

Rosenblueth, E (1992), "The social value of human life in earthquake engineering", International Symposium on Earthquake Disaster Prevention, CENAPRED, México DF, Mexico, II.

Starr, C (1969), "Societal benefit versus technological risk", Science, 165, 1232-48.

Starr, C y C Whipple (1980), "Risk of risk decisions", Science, 208, 1114-19.

Vargas E, Y JM Jara JM (1989), "Influencia del coeficiente sísmico de diseño en el costo de edificios con marcos de concreto", Memorias del VIII congreso nacional de ingeniería sísmica y VII congreso nacional de ingeniería estructural. Acapulco, Gro., nov.

von Neumann, J. y O Morgenstern (1943). Theory of games and economic behavior, Princeton University Press, Princeton, New Jersey; $2^{\text {nd }}$ ed, 1953.

Whitman RV, JM Biggs, J Brennan III, CA Cornell, RY de Neufville, E Vanmarcke (1973), "Summary of methodology and pilot application, seismic decision analysis", reporte no. 9, Department of Civil Engineering, MIT. 